\title{
Common universal behavior of magnetic domain walls driven by spin-polarized electrical current and magnetic field
}

\author{
R. Díaz Pardo, ${ }^{1}$ N. Moisan, ${ }^{1}$ L. J. Albornoz,,${ }^{1,2,3}$ A. Lemaître, ${ }^{4}$ J. Curiale, ${ }^{2,3}$ and V. Jeudy $\odot^{1, *}$ \\ ${ }^{1}$ Laboratoire de Physique des Solides, Université Paris-Sud, Université Paris-Saclay, CNRS, UMR8502, 91405 Orsay, France \\ ${ }^{2}$ Instituto de Nanociencia y Nanotecnología CNEA-CONICET, Centro Atómico Bariloche, Av. Bustillo 9500, \\ R8402AGP, San Carlos de Bariloche, Argentina \\ ${ }^{3}$ Instituto Balseiro, Universidad Nacional de Cuyo - CNEA, Av. Bustillo 9500, R8402AGP, S. C. de Bariloche, Rio Negro, Argentina \\ ${ }^{4}$ Centre de Nanosciences et de Nanotechnologies (C2N), CNRS, Université Paris-Sud, Université Paris-Saclay, 91120 Palaiseau, France
}

(Received 25 April 2019; revised manuscript received 25 October 2019; published 25 November 2019)

\begin{abstract}
We explore the universal behaviors of a magnetic domain wall driven by the spin-transfer torque of an electrical current, in a ferromagnetic $(\mathrm{Ga}, \mathrm{Mn})(\mathrm{As}, \mathrm{P})$ thin film with perpendicular magnetic anisotropy. For a current transverse to the domain wall, the dynamics of the thermally activated creep regime and the depinning transition are found to be compatible with a self-consistent universal description of magnetic-fieldinduced domain-wall dynamics. This common universal behavior, characteristic of the so-called quenched Edwards-Wilkinson universality class, is confirmed by an independent analysis of domain-wall roughness. Complementary investigations reveal the directional properties of interaction between current and domain walls which result in the instability of their transverse orientation.
\end{abstract}

DOI: 10.1103/PhysRevB.100.184420

\section{INTRODUCTION}

The displacement of small spin textures such as magnetic domain walls (DWs) thanks to spin torque effects is at the basis of potential applications of magnetic data storage [1]. An important effort is ongoing to find magnetic materials $[2,3]$ with large and well-controlled DW velocities. However, DWs are very sensitive to weak pinning defects [4,5], which strongly reduce their mobility and produce roughening and stochastic avalanchelike motion [6,7]. Therefore, it is particularly interesting to better understand the contribution of pinning to current-induced DW dynamics.

Magnetic domain walls [4-12] present surprising universal critical behaviors, shared with a wide variety of moving interfaces such as the reaction front propagation in disordered flows [13], growing bacterial colonies [14], wetting [15], and motion of ferroelectric domain walls [16], to name a few. The interfaces are rough with self-affine width growing as $L^{\zeta}$, where $L$ the distance between two points of the interface and $\zeta$ the roughness exponent. Moreover, a depinning threshold force $f_{d}$ separates the so-called creep $\left(f<f_{d}\right)$ and depinning $\left(f \gtrsim f_{d}\right)$ regimes. In the creep regime, the velocity varies as an Arrhenius law $v \sim e^{-\Delta E / k_{B} T}[4,11,17]$, where $k_{B} T$ is the thermal fluctuation energy. $\Delta E$ is the effective pinning energy barrier height, which follows a universal power-law variation with the driving force $\Delta E \sim f^{-\mu}$, where $\mu$ is the creep exponent. In the depinning regime $[12,18,19]$, the effective pinning barriers are collapsed. The velocity presents power-law variations with drive $f$ and temperature $T: v \sim$ $\left(f-f_{d}\right)^{\beta}$ and $v\left(f_{d}\right) \sim T^{\psi}$, where $\beta$ and $\psi$ are the depinning and thermal rounding exponents, respectively.

\footnotetext{
*vincent.jeudy@u-psud.fr
}

Universal behaviors have been extensively investigated for DWs driven by magnetic field $(f \propto H)$ in ferromagnetic ultrathin films. For a large variety of materials, the measured values of the creep $(\mu=1 / 4)[4,9-11]$ and roughness $(\zeta \approx 0.66$ $[4,5,9]$ and $\zeta \approx 1.25$ [7]) exponents are compatible with the prediction for the motion of an elastic one-dimensional (1D) line in a short-range weak pinning disorder, described by the quenched Edwards-Wilkinson (qEW) universality class, with [20] and without [17,21] anharmonic contributions. Moreover, it was recently shown [12] that the depinning transition is compatible with the predictions for the $\mathrm{qEW}$ universality class $(\beta=0.25[18]$ and $\psi=0.15$ [19]).

In contrast, the universal behaviors of DW motion induced by spin-polarized electric current are more contentious. To the best of our knowledge, the universal behavior of the depinning transition has not yet been explored. For the creep motion, a compatibility with $\mu=1 / 4$ is suggested for DW driven by the conventional spin-transfer torque (STT) in $\mathrm{Pt} / \mathrm{Co} / \mathrm{Pt}$ nanowires [22] and by spin-orbit torque (SOT) in ferrimagnets [2]. However, rather intriguing differences between currentand magnetic-field-driven motion are also reported in the literature. Different values of the creep exponent were reported for other materials $(\mu=0.33 \pm 0.06$ for $(\mathrm{Ga}, \mathrm{Mn}) \mathrm{As}$ [8] and $\mu=0.39 \pm 0.06$ for $\mathrm{Ta} / \mathrm{CoFeB} / \mathrm{MgO}$ [10]), which are difficult to interpret. The tilting and faceting [5,23] of DWs, produced by the current, could suggest a compatibility with the quenched Kardar-Parisi-Zhang (qKPZ) universality class [5]. However, in the direction perpendicular to DW, the roughness is characterized by an exponent $\left(\zeta_{j}=0.69 \pm 0.04\right)$ independent of DW tilting angle and compatible with the measurement obtained for field-driven DW motion $\left(\zeta_{H}=\right.$ $0.68 \pm 0.04)$, while a different value $\left(\zeta_{j}=0.99 \pm 0.01\right)$ is obtained in the direction of current. Therefore, whether distinct or common universality classes describe the motion of 
DWs produced by current and magnetic field remains an open question. Moreover, the origin of STT-induced DW tilting and its contribution to DW dynamics and universal behaviors are not well understood.

In this paper, we propose an extensive and comparative study on DW motion driven by STT and magnetic field. Investigations were conducted with the ferromagnetic semiconductor (Ga,Mn)As, which presents a sufficiently low depinning threshold [24] to cover the creep, depinning, and flow dynamical regimes [25], and to discuss, in particular, DW tilting without pinning. Our stringent test of universality relies on the verification that magnetic-field-driven DW motion in our sample shares the common universal (material and temperature independent) behaviors encountered in a variety of other magnetic materials [4,5,9-11] and on the independent analysis of DW dynamics and roughness, which are both consistent with the conclusion of common universal behaviors for STT and field-driven DW motion.

The paper is organized as follows. After a description of the experimental techniques (Sec. II), we compare the shape evolution of DWs driven by STT and magnetic field and analyze their roughness (Sec. III). Section IV details a comparative study of DW dynamics. In Sec. V, we discuss the origin of DW faceting produced by STT and its implications for DW dynamics.

\section{EXPERIMENTAL TECHNIQUES}

The experiments were performed with rectangles of a 4-nm-thick (Ga,Mn)(As,P)/(Ga,Mn)As bilayer film patterned by lithography. The film was grown on a (001) GaAs/AlAs buffer [26]. It has an effective perpendicular anisotropy and a Curie temperature $\left(T_{c}\right)$ of $65 \mathrm{~K}$. The sizes of rectangles were $133 \times 210,228 \times 302$, and $323 \times 399 \mu \mathrm{m}^{2}$ (see Appendix A for the details). Two $40-\mu \mathrm{m}$-wide gold electrodes (separated by 110,204 , and $300 \mu \mathrm{m}$, respectively) were deposited by evaporation parallel to the narrow sides of rectangles. They were used to generate a homogeneous current density driving DW motion by STT. The pulse amplitude varied between 0 and $11 \mathrm{GA} / \mathrm{m}^{2}$. We verified that the Joule effect had a negligible contribution on DW dynamics (see Appendix B). Perpendicular magnetic field pulses of adjustable amplitude (0-65 mT) were produced by a 75-turn small coil (diameter $\sim 1 \mathrm{~mm}$ ) mounted on the sample. The DW displacement is observed using a magneto-optical Kerr microscope (resolution $\sim 1 \mu \mathrm{m}$ ). The DW velocity is defined as the ratio between the average displacement $\langle u\rangle$ and the pulse duration $\Delta t$, which varies between $1 \mu \mathrm{s}$ and $120 \mathrm{~s}$. The setup was placed against the cold finger of an optical He-flow cryostat, which allowed the temperature to be stabilized to between $5.7 \mathrm{~K}$ and $T_{c}$.

\section{DOMAIN-WALL DISPLACEMENT AND ROUGHNESS IN THE CREEP REGIME}

The time evolution of an initially almost flat DW driven by magnetic field and STT is compared in Fig. 1. For fielddriven DW motion, the average successive displacements are relatively similar. The initial DW shape is almost conserved during the motion. The DWs become sometimes strongly pinned and curved [see Fig. 1(a)] but are flattened again when

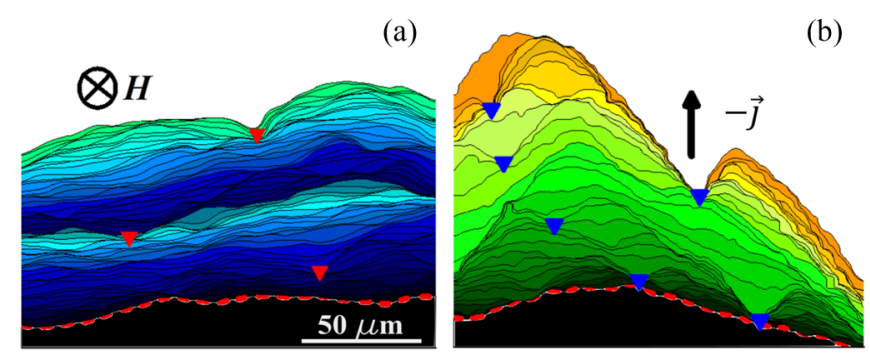

FIG. 1. Time evolution of DW shape. Successive positions of a DW, at $T=28 \mathrm{~K}$, driven by (a) magnetic field ( $H=0.16 \mathrm{mT}$, delay between images $\Delta t=0.5 \mathrm{~s}$, total duration $60 \mathrm{~s}$ ) and (b) current $\left(j \approx 0.5 \mathrm{GA} / \mathrm{m}^{2}, \Delta t=0.5 \mathrm{~s}\right.$, total duration $\left.16 \mathrm{~s}\right)$ observed at the same sample location. The DW move in the direction opposite to the current density, which is indicated by the arrow. The initial DW position is underlined by a thick dashed line. The triangles indicate the strongest DW pinning positions.

depinned due to the combined effects of DW elasticity and driving force, which acts as a pressure $(f \propto H)$. In contrast, the initial DW shape is significantly altered by the current [see Fig. 1(b)]. DWs are tilted and form faceted structures. The faceting process seems to start close to "strong" pinning centers [5] and to produce a reduction of DW displacements with increasing tilting angle $\theta$ until DWs stop (on the experiment timescale), as already observed in $\mathrm{Pt} / \mathrm{Co} / \mathrm{Pt}$ films [5]. In Sec. V, we will show that the faceting results from an instability of the transverse alignment between current and the DW, which can be triggered even without any contribution from pinning.

Let us start with investigations of universal behaviors with a study on DW self-affinity using the displacementdisplacement correlation function [4]:

$$
w(L)=\sum_{x}[u(x+L)-u(x)]^{2},
$$

where $u(x)$ is the DW displacement measured parallel to the current and $L$ the length of DW segment along the $x$ axis transverse to the current [see Fig. 2(a)]. For a self-affine interface, the function $w(L)$ is expected to follow a power-law variation $w(L) \sim L^{2 \zeta}$, where $\zeta$ is the roughness exponent. Typical variations of $w$ vs $L$ obtained for field- and current-induced motion are compared in Fig. 2(b) in log-log scale. As it can be observed, both curves present a linear variation with similar slopes $(=2 \zeta)$, between the microscope resolution $(\approx 1 \mu \mathrm{m})$ and $L=10 \mu \mathrm{m}$. In order to get more statistics, the slopes were systematically determined for successions of DW positions and a temperature varying over one decade $(T=4.5-59 \mathrm{~K}$, see Appendix $\mathrm{C}$ for the statistical study). The mean and standard deviation of the roughness exponent for current $\left(\zeta_{j}\right)$ - and field $\left(\zeta_{H}\right)$-driven DW motion is reported in Fig. 2 as a function of temperature. As expected for universal critical exponents, the values of $\zeta_{j}$ and $\zeta_{H}$ do not vary significantly. Their mean values $\left(\zeta_{j}=0.60 \pm 0.05\right.$ and $\left.\zeta_{H}=0.61 \pm 0.04\right)$, calculated from all measurements, agree well within experimental error, which is a signature of common universal behavior.

Here, it is important to discuss differences and similarities with the results reported for $\mathrm{Pt} / \mathrm{Co} / \mathrm{Pt}$ in Ref. [5]. For $(\mathrm{Ga}, \mathrm{Mn})(\mathrm{As}, \mathrm{P}), \zeta_{j}$ presents no significant variation 


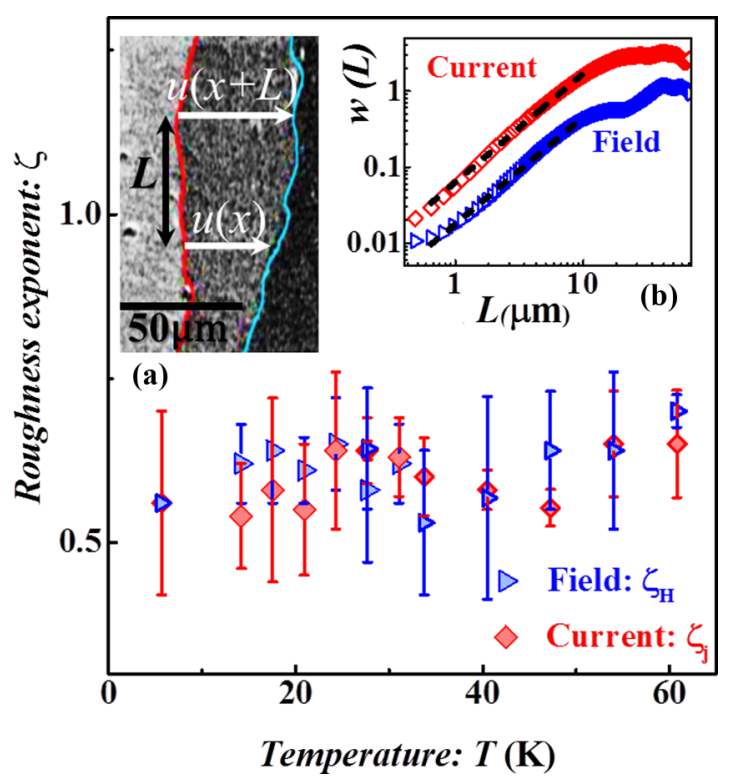

FIG. 2. Analysis of domain-wall roughness. Roughness exponents $\zeta_{j}$ and $\zeta_{H}$ as a function of temperature, measured for $j<j_{d}$ and $H<H_{d}$, respectively. Inset (a): Definition of the domain-wall displacements $u(x)$ and $u(x+L)$ used to calculate the displacementdisplacement correlation function [see Eq. (1)]. Inset (b): Typical correlation functions $w$ vs DW segment length $L$ in log-log scale for current-driven (diamonds) and field-driven (triangles) DWs ( $T=$ $14.2 \mathrm{~K})$. The dashed lines are a fit of $w \sim L^{2 \zeta}$ used to determine the roughness exponents $\zeta_{j}$ and $\zeta_{H}$.

with DW tilting and remains significantly smaller than the value $\left(\zeta_{j}=0.99 \pm 0.01\right)$ reported for DW displacements measured in the direction of current. A value $\zeta_{j} \approx 1$ most probably reflects the large linear tilting trend of DWs [27] [inserting a linear variation of $u(x)$ in Eq. (1) leads to $\zeta=1$ ] and not a universal behavior of their roughness. In contrast, the value $\zeta_{j}=0.60 \pm 0.05$ is compatible with the results $\left(\zeta_{j}=0.68 \pm 0.04\right)$ of Ref. [5] for a DW roughness analyzed in the direction $(\vec{n})$ normal to DW.

Therefore, the compatibility of results obtained for $(\mathrm{Ga}, \mathrm{Mn})(\mathrm{As}, \mathrm{P})$ and $\mathrm{Pt} / \mathrm{Co} / \mathrm{Pt}$ films [5] strongly suggests that the common universal behaviors for current- and field-driven DW motion should be observed in a variety of other materials. Interestingly, as the equilibrium roughness $\zeta(\approx 2 / 3)$ and the creep exponents are linked by the scaling relation $[4,17]$ $\mu=(2 \zeta-1) /(2-\zeta)$, it is natural to infer that the creep motion of DW driven by STT should be characterized by the same universal exponent $(\mu=1 / 4)$ as the magnetic-fielddriven DW motion, in agreement with the results obtained for $\mathrm{Pt} / \mathrm{Co} / \mathrm{Pt}$ nanowires [22]. Moreover, the depinning transition should also be described by the values of universal exponents $\beta(=0.25)$ and $\psi(=0.15)$ expected for the qEW universality class.

\section{DOMAIN-WALL DYNAMICS}

In order to discuss those assertions, we have investigated the DW dynamics. The velocity measurements were all performed with almost flat DWs transverse to the current $(\theta \approx 0)$ (see Fig. 1) in order to circumvent the variation of driving

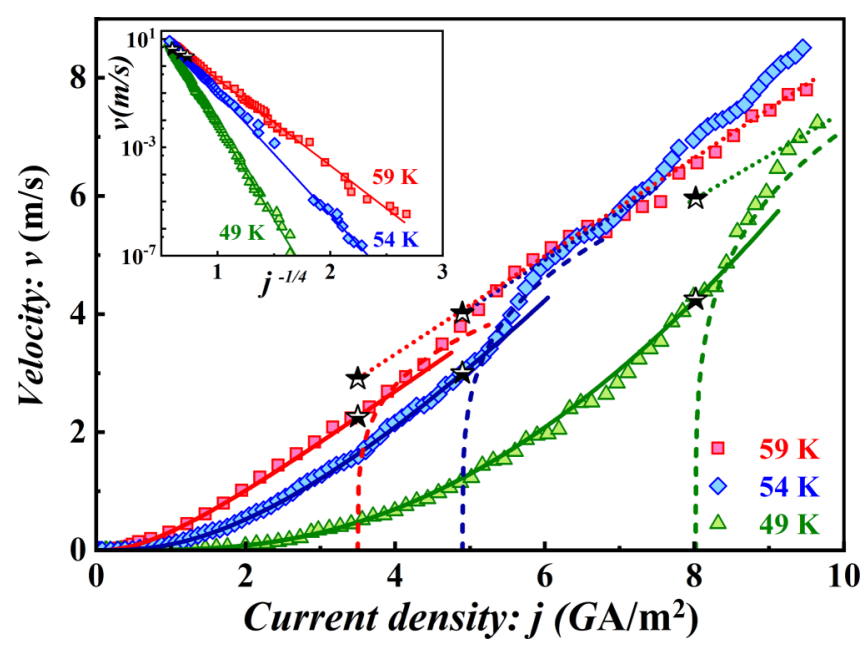

FIG. 3. STT-induced domain-wall dynamics. DW velocity vs current density $j$ for different temperatures. The solid, dashed, and dotted lines are predictions for the creep, depinning, and flow regimes, respectively, obtained from simultaneous fits of Eqs. (2). The lower and upper stars correspond to the velocities at the depinning threshold $v\left(j_{d}\right)$ and $v_{T}\left(j_{d}\right)$, respectively. A sliding average over five points is used to smooth the curves. The unexpected large velocities measured for $T=54 \mathrm{~K}$ and $j>7.5 \mathrm{GA} / \mathrm{m}^{2}$ were not used for the fit. Inset: Semilog plot of the same velocity curves vs $j^{-1 / 4}$ highlighting the thermally activated creep regime and its fit.

force with DW tilt. The velocity curves are reported in Fig. 3 as well as their analysis using a self-consistent description of the universal behaviors developed for field-driven DW motion $[12,24,28-30]$ :

$$
v(j)=\left\{\begin{array}{lr}
v\left(j_{d}\right) \exp \left(-\frac{\Delta E}{k_{B} T}\right) & \text { creep }: j<j_{d} \\
\frac{v_{T}\left(j_{d}\right)}{x_{0}}\left(\frac{j-j_{d}}{j_{d}}\right)^{\beta} & \text { depinning }: j \gtrsim j_{d} \\
v_{T}\left(j_{d}\right) \frac{j}{j_{d}} & \text { linearflow }: j \gg j_{d},
\end{array}\right.
$$

where $j_{d}$ is the depinning threshold and $v\left(j_{d}\right)$ the velocity at the depinning threshold. For the thermally activated creep regime, the energy barrier height is given by $\Delta E=$ $k_{B} T_{d}\left[\left(j / j_{d}\right)^{-\mu}-1\right]$, where $k_{B} T_{d}$ is the characteristic height of the effective pinning barrier. For the linear flow regime, $v_{T}\left(j_{d}\right)=v\left(j_{d}\right)\left(T_{d} / T\right)^{\psi}$ is the velocity that DW would reach at $j=j_{d}$ without pinning. The description of the depinning regime in Eqs. (2) is only valid over a limited range: it does not account for the effect of thermal fluctuations occurring at and just above the depinning threshold nor for the crossover to the linear flow regime [12]. Note that the critical exponents $\mu(=1 / 4), \beta(=0.25)$, and $\psi(=0.15)$, and the parameter $x_{0}(=0.65)$ in Eqs. (2) characterize the universal (i.e., temperature and material independent) behaviors of fielddriven DW motion, which are described by the qEW minimal model [12,17-19]. Therefore, the comparison between the velocity curves (Fig. 3) and Eqs. (2) relies only on three adjustable temperature- and material-dependent parameters [ $j_{d}(T), v\left(j_{d}(T)\right)$, and $\left.T_{d}(T)\right]$, whose values are related to the micromagnetic and microscopic pinning parameters [24].

To evidence the depinning threshold, we performed fits of the creep law for increasing values of $j_{d}(T)$ and $v\left(j_{d}, T\right)$ on the velocity curves $\left[T_{d}(T)\right.$ is the free parameter]. As it can be 
TABLE I. Pinning parameters of DW dynamics and fitting parameters of Eqs. (2). Units: $T$ and $T_{d}$ are in Kelvin, $v\left(j_{d}\right)$ and $v\left(H_{d}\right)$ in $\mathrm{m} / \mathrm{s}, j_{d}$ in $\mathrm{GA} / \mathrm{m}^{2}$, and $H_{d}$ in $\mathrm{mT}$. The numbers in parenthesis indicate the error bars.

\begin{tabular}{lccccccc}
\hline \hline & \multicolumn{3}{c}{ Current } & & \multicolumn{3}{c}{ Magnetic field } \\
\cline { 2 - 5 } \cline { 6 - 8 }$T$ & $j_{d}$ & $v\left(j_{d}\right)$ & $T_{d}$ & & $H_{d}$ & $v\left(H_{d}\right)$ & $T_{d}$ \\
\hline 49 & $7.9(0.6)$ & $4.1(0.5)$ & $465(35)$ & & $28.4(0.4)$ & $7.5(0.4)$ & $320(15)$ \\
54 & $4.9(0.4)$ & $3.0(0.4)$ & $375(25)$ & & $15.5(0.4)$ & $4.0(0.2)$ & $420(20)$ \\
59 & $3.5(0.3)$ & $2.3(0.2)$ & $315(30)$ & & $16.1(0.5)$ & $4.2(0.4)$ & $410(20)$ \\
\hline \hline
\end{tabular}

observed in Fig. 3 and its inset, the creep law describes well the experimental data from the lowest velocity $\left(v \approx 10^{-7} \mathrm{~m} / \mathrm{s}\right)$ up to limits $\left(j \approx 8,5\right.$, and $3.5 \mathrm{GA} / \mathrm{m}^{2}$ for $T=49,54$, and $59 \mathrm{~K}$, respectively) above which a systematic disagreement is observed. In order to verify that those limits are effectively signatures of the depinning threshold, we performed simultaneous fits of the predictions of Eq. (1) for the depinning and flow regime using a fine tuning of point $\left[j_{d}(T), v\left(j_{d}, T\right)\right]$ on the creep law.

The coordinates of optimum points (indicated by stars with half filled down in Fig. 3) and the values of $T_{d}(T)$, obtained at different temperatures, are reported in Table I. The good agreement between the velocity curves and the predictions of Eqs. (2) evidences the depinning threshold and demonstrates that the observed DW dynamics covers the creep, depinning, and flow regimes. Note that the investigation of DW motion above the depinning threshold was not accessible in experiments with $\mathrm{CoFeB}[10]$ and even less with $\mathrm{Pt} / \mathrm{Co} / \mathrm{Pt}[5,22]$ due to the too low efficiency of STT.

The compatibility of DW dynamics with Eqs. (2) is a strong evidence that DW motion induced by transverse current through the STT mechanism shares the common universal behavior of DW driven by a magnetic field observed in thin magnetic films made of different materials [4,9-12,24]. Our analysis of DW dynamics and roughness is compatible with the conclusions reported for the creep motion in $\mathrm{Pt} / \mathrm{Co} / \mathrm{Pt}$ films [5,22]. In contrast, while a similar material is investigated, the results obtained for $(\mathrm{Ga}, \mathrm{Mn})$ As nanowires [8] are not confirmed (see also the Supplemental Material of Ref. [11] for field-driven DW motion over a large velocity range). The different values of creep exponent reported in Refs. [8] and [10] could originate from a pinning of DWs by the edges of nanowires [28] or from the tilting of DW, which is not controlled.

Beyond the analysis of universal behavior, it is also particularly interesting to compare the material-dependent pinning properties of DWs driven by STT and magnetic field. To achieve this objective, we have performed the analysis of magnetic-field-driven DW dynamics close to the depinning transition (see Fig. 4). For the fit of velocity curves we used the procedure described above $\left[j_{d}(T)\right.$ was replaced by $H_{d}(T)$ ]. The pinning parameters are reported in Table I. Following Ref. [22], we can define the fieldlike effect of the STT by $H_{d}=\epsilon J_{d}$. The obtained value $\left(\epsilon \approx 4 \times 10^{-12} \mathrm{~T} \mathrm{~m}^{2} / A\right)$ is more than 2 orders of magnitude larger than for $\mathrm{Pt} / \mathrm{Co} / \mathrm{Pt}$ films $\left(=1.6 \times 10^{-14} \mathrm{~T} \mathrm{~m}^{2} / A\right)$ [22], reflecting the much larger efficiency of STT in $(\mathrm{Ga}, \mathrm{Mn})(\mathrm{As}, \mathrm{P})$. In addition, the similar values of $T_{d}$ obtained for field- and current-driven DW

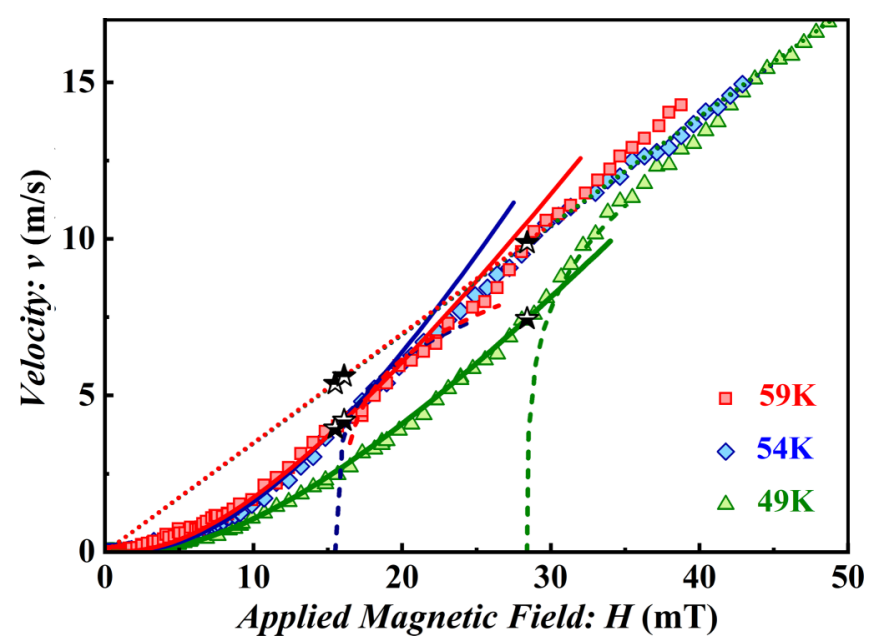

FIG. 4. Magnetic-field-induced domain-wall dynamics. DW velocity vs magnetic field $H$ for different temperatures and their fit with Eqs. (2). The solid, dashed, and dotted lines are predictions for the creep $\left[H<H_{d}(T)\right]$, depinning $\left[H \gtrsim H_{d}(T)\right]$, and flow $[H \gg$ $\left.H_{d}(T)\right]$ regime, respectively. The lower and upper stars correspond to the velocities $v\left(H_{d}\right)$ and $v_{T}\left(H_{d}\right)$ at the depinning threshold $H_{d}$, respectively. The slightly nonlinear variations observed for $T=59 \mathrm{~K}$ and $H>33 \mathrm{mT}$ were not used for the fit of flow regime.

dynamics $\left[T_{d}(H) / T_{d}(j)=1.0 \pm 0.3\right]$ indicate that a similar random disorder controls DW pinning. Finally, the ratio $T_{d} / T$ $(\approx 5-10)$ is rather small compared to other materials [12,24], which explains why the depinning threshold is not straightforward to observe in the velocity curves (Figs. 3 and 4) without a comparison with the fit of Eqs. (2).

\section{ORIGIN OF DOMAIN-WALL FACETING}

Let us now discuss the tilting and faceting of DWs, which is observed only for current-driven motion (see Fig. 1 and Ref. [5]). First, we analyze the evolution of an initially almost rectangular domain subjected to a current density $\left(j=11 \mathrm{GA} / \mathrm{m}^{2}\right)$, sufficiently large to move untilted DWs $(\theta=0$, i.e., $\vec{j} \| \vec{n}$, where $\vec{n}$ is the direction normal to DW) in the flow regime. As it can be observed in Fig. 5, the edges of the domain aligned along the current (i.e., $\vec{j} \perp \vec{n}$ ) remain almost motionless. In contrast, the back and front DWs perpendicular to the current (i.e., $\vec{j} \| \vec{n}$ ) are significantly displaced. Surprisingly, the back DW moves faster than the front DW, which causes the collapse of the domain [see Figs. 5(e)-5(f)]. Another interesting feature is the increasingly pointed shape of the front DW (not observed for the back DW). Here, the faceting of the front DW develops without any contribution of "strong" pinning sites, which suggests that the transverse orientation between DW and current is unstable. Consequently, the different shape evolutions of the front and back DWs observed in Figs. 5(a)-5(f) can be interpreted as a result of opposite contributions of DW elasticity. The two side DWs pull the extremities of the back (front) DW in the $-\vec{j}(\vec{j})$ direction, which tends to stabilize (destabilize) the transverse DW orientation.

To explore more quantitatively the directionality of interaction between DW and current, we have measured the DW 

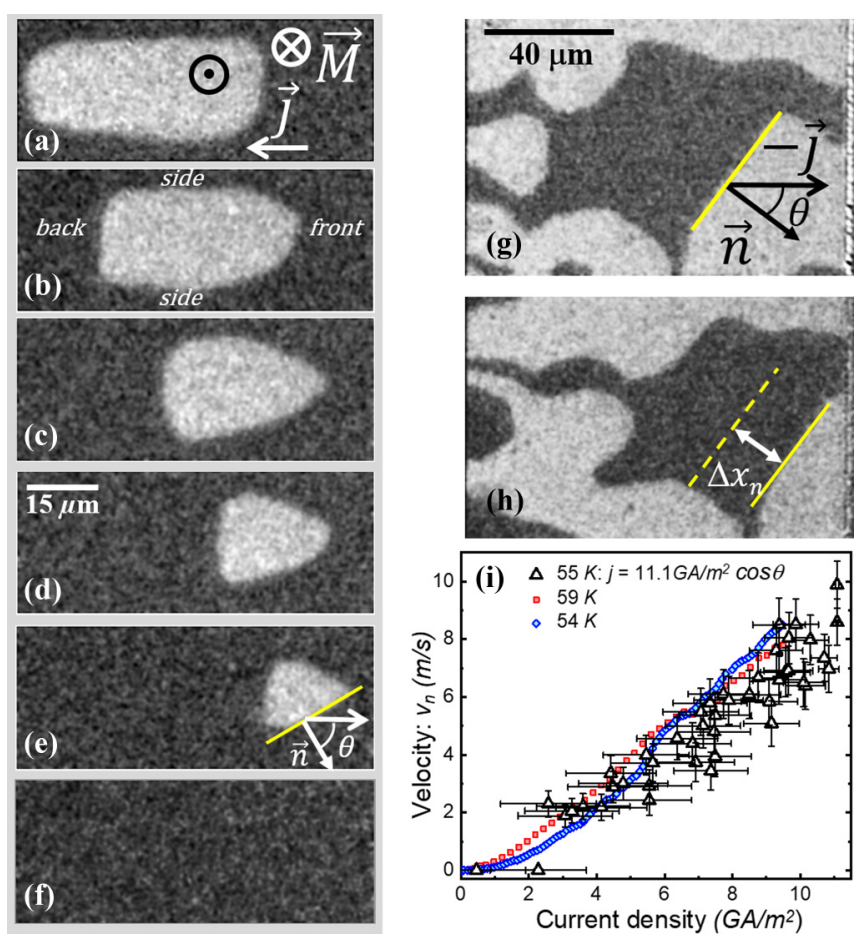

FIG. 5. Current-driven domain-wall motion. (a-f) Evolution of an almost rectangular magnetic domain submitted to pulses of current (duration $\Delta t=6 \mu \mathrm{s}$ and amplitude $j=11 \mathrm{GA} / \mathrm{m}^{2}$ ) for $T=$ $45 \mathrm{~K}$. The gray levels correspond to opposite directions of magnetization $\vec{M}$ perpendicular to the sample. The back DW conserves its shape and velocity while the front DW becomes pointed, which reduces its velocity and leads to the collapse of the domain (see images e and $\mathrm{f}) .(\mathrm{g}, \mathrm{h})$ Two successive images of domains $(\Delta t=1 \mu \mathrm{s}$ and $j=11.1 \mathrm{GA} / \mathrm{m}^{2}$ ) showing, for $T=55 \mathrm{~K}$, the displacement $\Delta x_{n}$ of a tilted DW (see the dashed and solid segments) along its normal direction $\vec{n}$. $\theta$ is the tilting angle between $\vec{n}$ and $-\vec{j}$. (i) Curve of the velocity $v_{n}$ along $\vec{n}$ vs $|j| \cos \theta$ obtained for a constant value of $j\left(=11.1 \mathrm{GA} / \mathrm{m}^{2}\right)$ for $T=55 \mathrm{~K}$, and $\Delta t=1 \mu \mathrm{s}$, and comparison with the velocity curves vs current density $(\theta=0)$ taken from Fig. 3. The error bars reflect the uncertainties of tilting angle and displacement.

displacements $\Delta x_{n}$ along direction $\vec{n}$ as a function of the angle $\theta$ between $\vec{n}$ and $-\vec{j}$ for a fixed magnitude of current density and pulse duration $\Delta t=1 \mu \mathrm{s}$ [see Figs. 5(g) and 5(h)]. We have then deduced the variation of velocity $v_{n}=\Delta x_{n} / \Delta t$ as a function of $|j| \cos \theta$, which is reported Fig. 5(i). As expected, the DW velocity decreases as the tilting angle increases (i.e., $|j| \cos \theta$ decreases). For $|j| \cos \theta<2.25 \mathrm{GA} / \mathrm{m}^{2}$, the displacement $\Delta x_{n}$ becomes lower than the spatial resolution so that the estimation for $v_{n}$ is zero. The dispersion of data most probably results from the contributions of DW elasticity, which tends to reduce DW velocity. Therefore, the velocity DW without contribution of elasticity should correspond to the upper measured values. Interestingly, those values present a rather good overlap with the velocity curves of Fig. 3, which were obtained as a function of the current density and for $\theta=0$. This indicates that DW tilting reduces the driving force in the direction normal $\vec{n}$ to the DW and suggests that the DW faceting originates from the directionality of the driving force $f \propto \vec{j} \cdot \vec{n}$.
Let us now discuss the consequence of the variation of the driving force with DW tilting $(\propto j \cos \theta)$ on universal behaviors. The second-order expansion of $\cos \theta[\approx 1-$ $\left.(1 / 2)(\partial u / \partial x)^{2}\right]$ introduces a so-called KPZ term $\left[\lambda(\partial u / \partial x)^{2}\right.$ with $\lambda=-j / 2$ ] in the equation of motion [31], which is usually used to describe interface tilting. However, at large tilting angle, the relation $f \propto \vec{j} \cdot \vec{n}$ should not be compatible with the KPZ minimal model. In contrast, in the direction $\vec{n}$ perpendicular to DW, for a fixed DW tilting, the driving force due to spin-transfer torque is simply proportional to $j \cos \theta$ and acts as a magnetic field $(f \propto H)$. This could explain the compatibility of current-induced DW motion with the qEW universality class, which is observed experimentally.

\section{CONCLUSION}

In conclusion, we have shown that the roughness and dynamics of DWs driven by spin-transfer torque and magnetic field present common universal behaviors compatible with a short-range weak pinning disorder described by the quenched Edwards-Wilkinson universality class. The agreement with the self-consistent description of creep and depinning regimes should help to better understand spin-transfer-torqueinduced DW motion experiments [2], since it allows clear identification of dynamical regimes and discrimination between universal and material-dependent behaviors [24]. In this frame, it would be also very interesting to study the universal behaviors of DWs driven by spin-orbit torque [32] and of DWs in antiferromagnets [33].

Moreover, we evidence the instability of transverse alignment between DW and current in the creep and flow regimes. The latter originates from the decrease of driving force and consequently of DW velocity with DW tilting. Our results also strongly suggest that for sufficiently large tilting angle, the motion of DWs is not compatible with the quenched KardarParisi-Zhang minimal model [31]. The contribution of DW tilting to their dynamics should also have direct implications for potential applications based on the controlled-motion DWs [1] in nanowires. Indeed, the spin-transfer torque tends to stabilize DW alignment in the direction of current.

\section{ACKNOWLEDGMENTS}

We wish to thank S. Bustingorry, A. Kolton, and K. Wiese for fruitful discussion, and J. Miltat and J. Sampaio for careful reading of the manuscript. This work was partly supported by the projects DIM CNano IdF (Region Ile-de-France) and the Labex NanoSaclay (ANR-10-LABX-0035). R.D.P. thanks the Mexican council CONACyT for the Ph.D. fellowship (No. 449563).

\section{APPENDIX A: SAMPLE DETAILS}

The devices used for our study are shown in Fig. 6(a). They consist of a 4-nm-thick (Ga,Mn)As/(Ga,Mn)(As,P) bilayer grown on a (001) GaAs/AlAs buffer and covered by a GaAs cap. The bilayer and the GaAs cap were grown at $\sim 220^{\circ} \mathrm{C}$, and the rest of the structure at $\sim 500{ }^{\circ} \mathrm{C}$ [26]. A postgrowth annealing was performed so as to remove interstitial $\mathrm{Mn}$ ions. The stack was then patterned by lithography in a set of rectangles with three different sizes: $133 \times 210,228 \times$ 


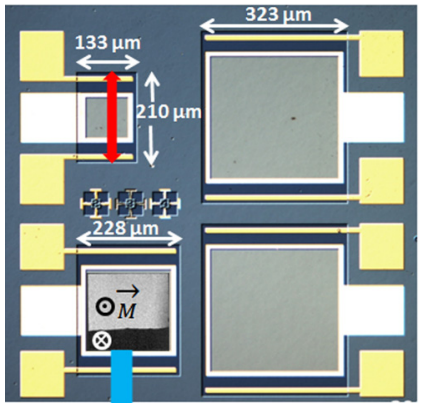

(a)

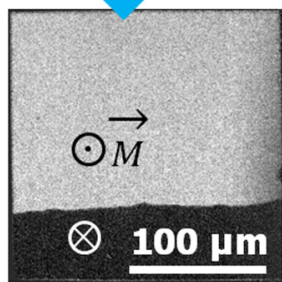

(c)

FIG. 6. Samples and velocity measurements. (a) Optical image of a set of devices showing the rectangular $(\mathrm{Ga}, \mathrm{Mn}) \mathrm{As} /(\mathrm{Ga}, \mathrm{Mn})(\mathrm{As}, \mathrm{P})$ bilayer of three different sizes, the connecting $\mathrm{Ti} / \mathrm{Au}$ electrodes (in yellow) used to generate current pulses, and an example of the location where domain-wall motion is observed. (b) Schematic of the stack. The direction of red arrow is the same as in (a). The DW motion is observed through the $\mathrm{SiO}_{2}$ and the thinnest part of the $\mathrm{Au} / \mathrm{Ti}$ electrode deposited above. The latter is not connected for the presented experiments. (c, d) Differential polar Kerr images showing two successive positions of a DW, which separates regions with opposite magnetization directions perpendicular to the bilayer. The displacement of the DW was produced by $0.45 \mathrm{GA} / \mathrm{m}^{2}$ current-density pulse of 10 -s duration. (e) Subtraction of images (d) and (c) highlighting the DW displacement $u(x)$.

302, and $323 \times 399 \mu \mathrm{m}^{2}$ [see Fig. 6(a)]. A 55-nm-thick $\mathrm{SiO}_{2}$ gate-oxide layer was deposited by plasma-enhanced chemical vapor deposition at $200{ }^{\circ} \mathrm{C}$ and then covered by an evaporated film of Ti/Au serving as the gate [26] [see Fig. 6(b)]. For the experiments presented here, this gate was not connected. The motion of domain walls (DWs) in the bilayer was observed through the square part of the Ti/Au gate [see the bottom left of Fig. 6(a)].

Finally, Ti $(20 \mathrm{~nm}) / \mathrm{Au}(200 \mathrm{~nm})$ electrode bars of width $40 \mu \mathrm{m}$ were deposited onto the GaAs cap on both sides of the rectangle to connect the ferromagnetic $(\mathrm{Ga}, \mathrm{Mn}) \mathrm{As} /(\mathrm{Ga}, \mathrm{Mn})(\mathrm{As}, \mathrm{P})$ bilayer. Those electrodes were used to generate a homogeneous current density in the direction of the largest length of rectangles. The current flows only within the (Ga,Mn)As/(Ga,Mn)(As,P) bilayer, since the embedding media are undoped GaAs layers with a much larger resistivity. Therefore, the motion of DW produced by the current is due to the spin-transfer torque (STT). The measurement of velocity was always performed from an initially almost flat magnetic domain wall aligned in the direction parallel to the $\mathrm{Ti} / \mathrm{Au}$ electrodes (i.e., perpendicular to direction of electric current), as shown in Figs. 6(a) and 6(c). The displacement of DW to a new position [see Fig. 6(d)] was produced by

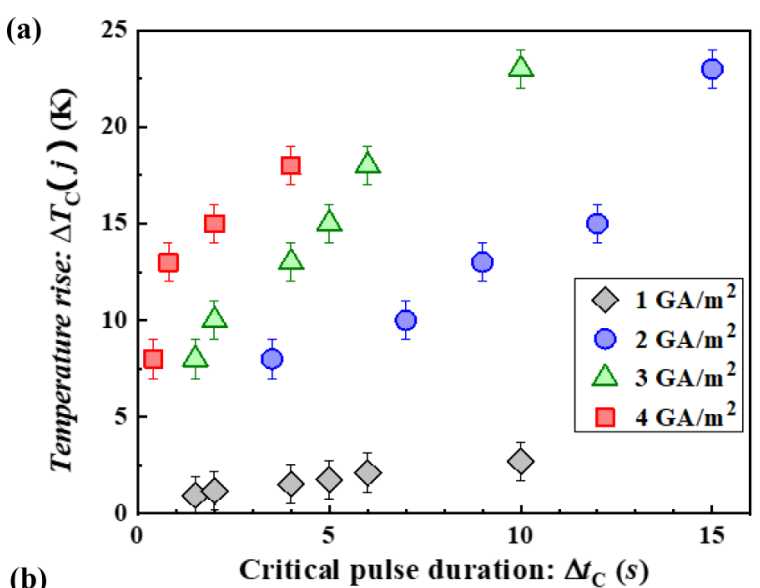

(b)

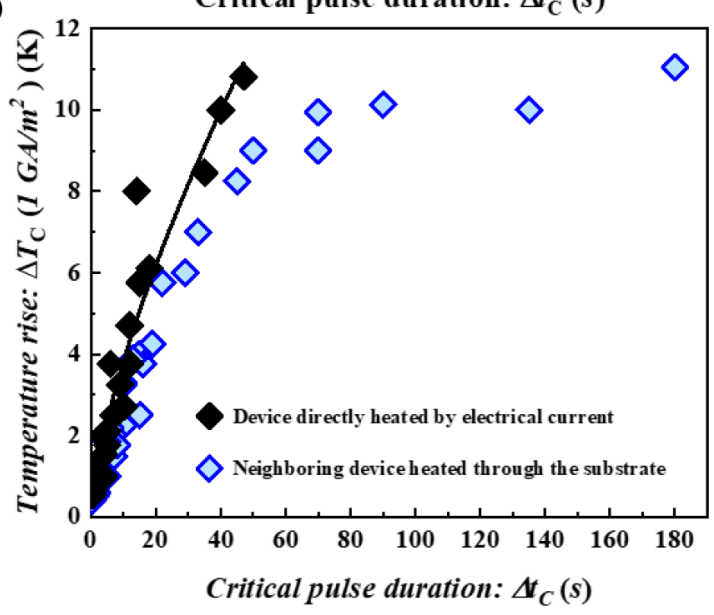

FIG. 7. Temperature rise produced by Joule heating. (a) Temperature rise vs time for different current densities. (b) Normalized temperature rise for a $1 \mathrm{GA} / \mathrm{m}^{2}$ pulse for the device $(228 \times$ $302 \mu \mathrm{m}^{2}$ ) connected to the current source (solid diamonds) and for a device with the same dimensions placed $\sim 1 \mathrm{~mm}$ away (empty diamonds). The solid line corresponds to the best fit of the function $\Delta T\left(t, 1 \mathrm{GA} / \mathrm{m}^{2}\right)=\mathrm{aT}^{\mathrm{b}}$ for $t<50 \mathrm{~s}$.

magnetic field pulses (amplitude from 0 to $65 \mathrm{mT}$ and duration $\Delta t=1 \mu \mathrm{s}$ up to $100 \mathrm{~s}$ ) or current pulses (amplitude from 0.02 to $9.70 \mathrm{GA} / \mathrm{m}^{2}$ and $\Delta t=1 \mu \mathrm{s}$ to $60 \mathrm{~s}$ ). The displacement of DW $u(x)$ was measured in the direction of current and could vary along the DW line $x$ (as observed in Figs. 6(e) and 2). The velocity is defined as the ratio between the average DW displacement and the pulse duration $[\langle u\rangle / \Delta t]$.

\section{APPENDIX B: CONTRIBUTION OF JOULE HEATING TO DW DYNAMICS}

In order to determine the temperature rise produced by current pulses and to confirm a negligible contribution of Joule heating to DW dynamics we have followed a similar analysis as that proposed in Ref. [34].

For each measurement, we prepare an initial magnetic state consisting in a closed magnetic domain surrounded by a homogeneous reversed magnetization. First, we determine the critical (Curie) temperature at which the 

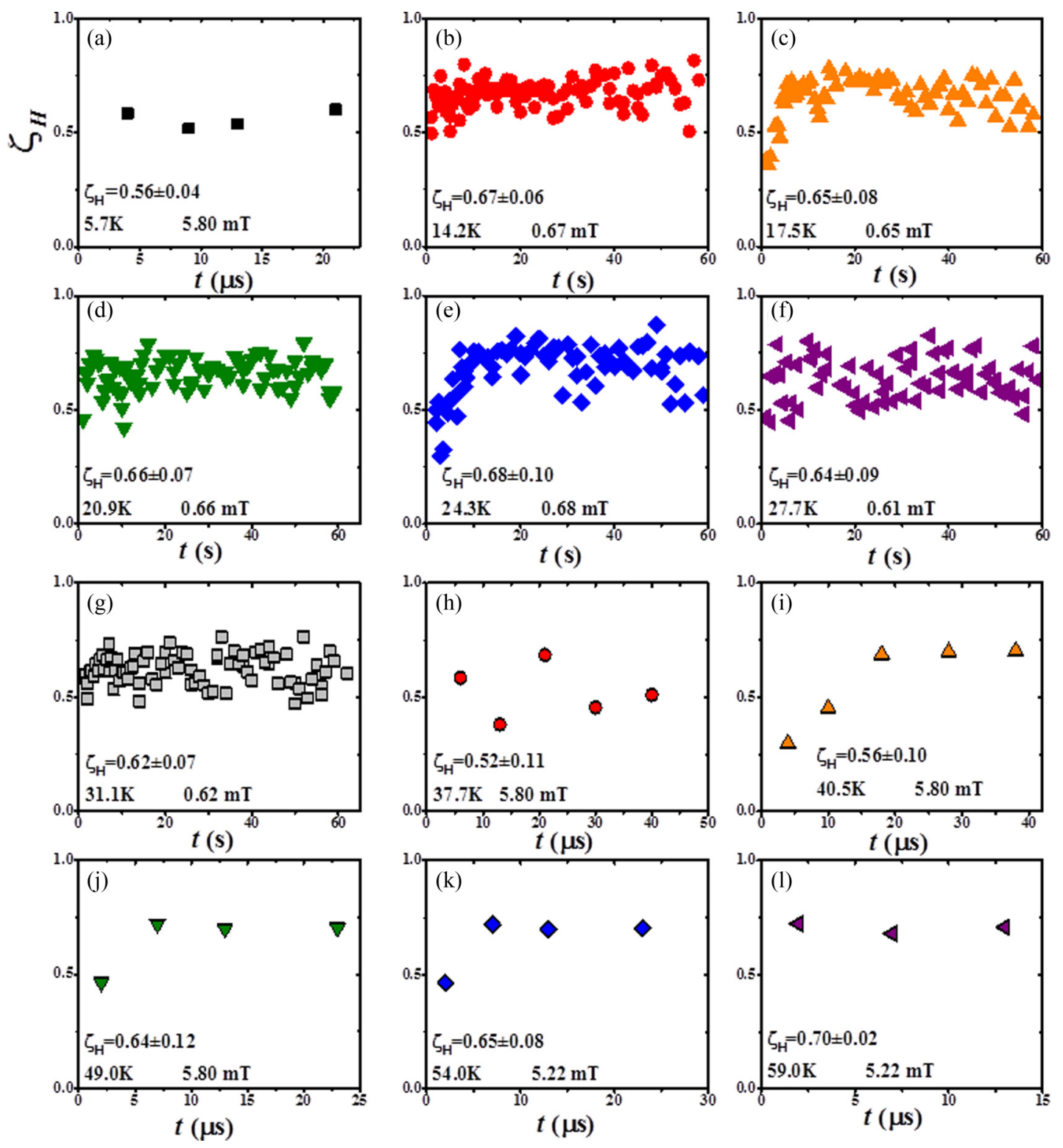

FIG. 8. Roughness exponent vs time for field-induced DW motion. (a)-(l) Temporal evolution of the roughness exponent at different temperatures and constant magnetic field amplitude within the creep regime. In every case $\frac{H}{H_{d}}<1$. The average $\zeta_{H}$ is indicated for each temperature.

contrast of the polar magneto-optical Kerr effect vanishes $\left(T_{c}=65 \mathrm{~K}\right)$ by progressively increasing the temperature. Then, the experimental protocol used for measuring the temperature rise $\Delta T$ produced by current pulses is as follows:

Step 1 . We set an initial sample temperature $T_{i}$. This fixes the temperature rise $\left(\Delta T_{c}=T_{c}-T_{i}\right)$ required to reach the temperature $T_{c}$.

Step 2. We choose a value for the current density $j$. We then apply a pulse of duration $\Delta t$ which produces a temperature rise $\Delta T(j, \Delta t)$. If $\Delta T(j, \Delta t)<\Delta T_{c}$, the magnetic domain is still visible after the pulse; the sample has remained in the ferromagnetic state during the pulse. In contrast, if $\Delta T(j, \Delta t)>\Delta T_{c}$, the Joule heating has produced a transition to the paramagnetic state and the domain is no longer observed after the current pulse. In order to estimate the value of the critical pulse duration $\Delta t_{c}$ for which $\Delta T\left(j, \Delta t_{c}\right)=$
$\Delta T_{c}$, we progressively increase the pulse duration until the vanishing of the magnetic domain.

Step 3. Step 2 is repeated for different values of the current density $j$ and the same temperature rise $\Delta T_{c}$.

Step 4. Steps 1-3 are repeated for different values of $\Delta T_{c}$.

For each value of current density, this procedure enables one to associate a temperature rise $\Delta T\left(j, \Delta t_{c}\right)$ to a pulse duration $\Delta t_{c}$ as shown in Fig. 7(a). In order to verify that the obtained curves reflect the contribution of Joule heating, the temperature rises were divided by $j^{2}$. As it can be observed in Fig. 7(b), all the data points collapse onto a single master curve, which confirms our assumption. The master curve corresponds to the time variation of the temperature rise $\Delta T$ produced by a current pulse of amplitude $j=1 \mathrm{GA} / \mathrm{m}^{2}$.

Moreover, the same type of measurement was performed for a device separated by $\sim 1 \mathrm{~mm}$ from the heating source. 

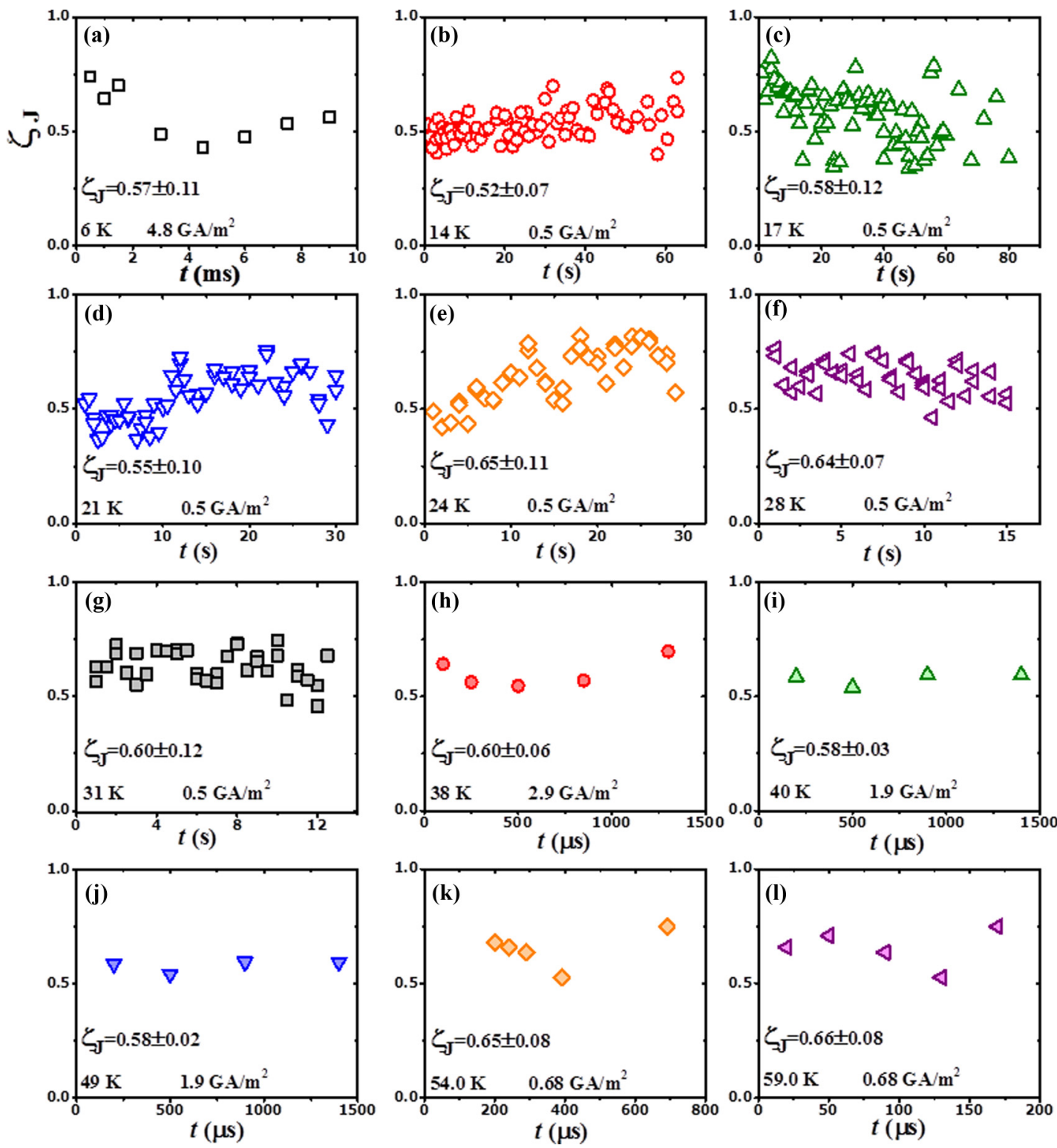

FIG. 9. Roughness exponent vs time for current-induced DW motion. (a)-(1) Temporal evolution of the roughness exponent at different temperatures and constant current density within the creep regime. In every case $\frac{j}{j_{d}}<1$. The average $\zeta_{J}$ is indicated for each temperature.

In this case, the heat is transferred through the substrate of bilayer devices. Therefore, the vanishing of the domain is assigned exclusively to temperature rise and cannot be associated to current-induced DW motion. As shown in Fig. 7(b), the obtained curve has a similar shape. The temperature rise presents a strong variation for the shortest duration $(t<50 \mathrm{~s})$. For $t>50 \mathrm{~s}$, the temperature rise tends to saturate, which probably reflects a quasithermal equilibrium controlled by the power produced by Joule heating, the cooling power of the cryostat, and the thermal impedance of the sample and substrate.

Let us now discuss the contribution of Joule heating to DW dynamics. We first seek for a continuous function describing the temperature rise for $\Delta t<50 \mathrm{~s}$. Arbitrarily, we have chosen a power-law variation $\Delta T\left(j=1 \mathrm{GA} / \mathrm{m}^{2}, \Delta \mathrm{t}\right)=$ $\mathrm{a}(\Delta \mathrm{t})^{\mathrm{b}}$ with $a=0.78 \pm 0.13$ and $b=0.69 \pm 0.05$, which fits the data [see Fig. 7(b)] rather accurately. Then, we have calculated the temperature rise for each pulse amplitude and duration used for the velocity measurements. The obtained maximum temperature rise is $\Delta T \leqslant 0.3 \mathrm{~K}$. The corresponding maximum velocity variation is $\frac{v(j, T)-v(j, T+0.3 \mathrm{~K})}{v(j, T)} \approx 3 \%$. A comparison with the log-log scale velocity curves presented in the main text for different temperatures directly shows that Joule heating produces an insignificant contribution to DW dynamics.

\section{APPENDIX C: STATISTICAL STUDY OF ROUGHNESS EXPONENT}

The roughness exponents for the magnetic field $\left(\zeta_{H}\right)$ - and current $\left(\zeta_{j}\right)$-driven DW wall were determined systematically from time sequences of DW displacement, similar to those shown in the Fig. 1 of the main text. Figures 8 and 9 show the time evolution of $\zeta_{H}$ and $\zeta_{j}$, respectively, for different 
temperatures in the range $5.7 \mathrm{~K}<T<59.0 \mathrm{~K}$. As it can be observed, $\zeta_{H}$ is found to be globally independent of time, except for some experiments [see Figs. 8(e), 8(i), and 8(j)] for which a variation is observed close to $t=0 \mathrm{~s}$. Similarly, $\zeta_{j}$ remains constant with time, which indicates that the faceting (tilting) of DWs observed in Fig. 1(b) produces no significant change of the measured value of $\zeta_{j}$ [see Fig. 9(f)]. The roughness exponent values $\zeta_{H}=0.61 \pm 0.03$ and $\zeta_{j}=0.60 \pm 0.05$ discussed in the paper are both averages over more than 350 measurements.
[1] S. S. P. Parkin and S.-H. Yang, Nat. Nanotechnol. 10, 195 (2015).

[2] L. Caretta, M. Mann, F. Büttner, K. Ueda, B. Pfau, C. M. Günther, P. Hessing, A. Churikova, C. Klose, M. Schneider, D. Engel, C. Marcus, D. Bono, K. Bagschik, S. Eisebitt, and G. S. D. Beach, Nat. Nanotechnol. 13, 1154 (2018).

[3] A. Hrabec, K. Shahbazi, T. A. Moore, E. Martinez, and C. H. Marrows, Nanotechnology 30, 234003 (2019).

[4] S. Lemerle, J. Ferré, C. Chappert, V. Mathet, T. Giamarchi, and P. Le Doussal, Phys. Rev. Lett. 80, 849 (1998).

[5] K.-W. Moon, D.-H. Kim, S.-C. Yoo, C.-G. Cho, S. Hwang, B. Kahng, B.-C. Min, K.-H. Shin, and S.-B. Choe, Phys. Rev. Lett. 110, 107203 (2013).

[6] E. E. Ferrero, L. Foini, T. Giamarchi, A. B. Kolton, and A. Rosso, Phys. Rev. Lett. 118, 147208 (2017).

[7] M. P. Grassi, A. B. Kolton, V. Jeudy, A. Mougin, S. Bustingorry, and J. Curiale, Phys. Rev. B 98, 224201 (2018).

[8] M. Yamanouchi, J. Ieda, F. Matsukura, S. E. Barnes, S. Maekawa, and H. Ohno, Science 317, 1726 (2007).

[9] P. J. Metaxas, J. P. Jamet, A. Mougin, M. Cormier, J. Ferré, V. Baltz, B. Rodmacq, B. Dieny, and R. L. Stamps, Phys. Rev. Lett. 99, 217208 (2007).

[10] S. DuttaGupta, S. Fukami, C. Zhang, H. Sato, M. Yamanouchi, F. Matsukura, and H. Ohno, Nat. Phys. 12, 333 (2016).

[11] V. Jeudy, A. Mougin, S. Bustingorry, W. Savero Torres, J. Gorchon, A. B. Kolton, A. Lemaître, and J.-P. Jamet, Phys. Rev. Lett. 117, 057201 (2016).

[12] R. Diaz Pardo, W. Savero Torres, A. B. Kolton, S. Bustingorry, and V. Jeudy, Phys. Rev. B 95, 184434 (2017).

[13] S. Atis, A. K. Dubey, D. Salin, L. Talon, P. Le Doussal, and K. J. Wiese, Phys. Rev. Lett. 114, 234502 (2015).

[14] J. A. Bonachela, C. D. Nadell, J. B. Xavier, and S. A. Levin, J. Stat. Phys. 144, 303 (2011).

[15] S. Moulinet, C. Guthmann, and E. Rolley, Eur. Phys. J. E 8, 437 (2002).

[16] T. Tybell, P. Paruch, T. Giamarchi, and J.-M. Triscone, Phys. Rev. Lett. 89, 097601 (2002).

[17] P. Chauve, T. Giamarchi, and P. Le Doussal, Phys. Rev. B 62, 6241 (2000).
[18] P. Le Doussal and K. J. Wiese, Phys. Rev. E 79, 051105 (2009).

[19] S. Bustingorry, A. B. Kolton, and T. Giamarchi, Phys. Rev. E 85, 021144 (2012).

[20] A. Rosso and W. Krauth, Phys. Rev. Lett. 87, 187002 (2001).

[21] A. B. Kolton, A. Rosso, T. Giamarchi, and W. Krauth, Phys. Rev. B 79, 184207 (2009).

[22] J.-C. Lee, K.-J. Kim, J. Ryu, K.-W. Moon, S.-J. Yun, G.-H. Gim, K.-S. Lee, K.-H. Shin, H.-W. Lee, and S.-B. Choe, Phys. Rev. Lett. 107, 067201 (2011).

[23] K.-W. Moon, C. Kim, J. Yoon, J. W. Choi, D.-O. Kim, K. M. Song, D. Kim, B. S. Chun, and C. Hwang, Nat. Commun. 9, 3788 (2018).

[24] V. Jeudy, R. Díaz Pardo, W. Savero Torres, S. Bustingorry, and A. B. Kolton, Phys. Rev. B 98, 054406 (2018).

[25] J. Curiale, A. Lemaître, C. Ulysse, G. Faini, and V. Jeudy, Phys. Rev. Lett. 108, 076604 (2012).

[26] T. Niazi, M. Cormier, D. Lucot, L. Largeau, V. Jeudy, J. Cibert, and A. Lemaître, Appl. Phys. Lett. 102, 122403 (2013).

[27] M. Höll, K. Kiyono, and H. Kantz, Phys. Rev. E 99, 033305 (2019).

[28] L. Herrera Diez, V. Jeudy, G. Durin, A. Casiraghi, Y. T. Liu, M. Voto, G. Agnus, D. Bouville, L. Vila, J. Langer, B. Ocker, L. Lopez-Diaz, and D. Ravelosona, Phys. Rev. B 98, 054417 (2018).

[29] K. Shahbazi, A. Hrabec, S. Moretti, M. B. Ward, T. A. Moore, V. Jeudy, E. Martinez, and C. H. Marrows, Phys. Rev. B 98, 214413 (2018).

[30] K. Shahbazi, J.-V. Kim, H. T. Nembach, J. M. Shaw, A. Bischof, M. D. Rossell, V. Jeudy, T. A. Moore, and C. H. Marrows, Phys. Rev. B 99, 094409 (2019).

[31] M. Kardar, G. Parisi, and Y.-C. Zhang, Phys. Rev. Lett. 56, 889 (1986).

[32] E. Martinez, S. Emori, and G. S. D. Beach, Appl. Phys. Lett. 103, 072406 (2013).

[33] S.-H. Yang, K.-S. Ryu, and S. Parkin, Nat. Nanotechnol. 10, 221 (2015).

[34] J. Curiale, A. Lemaître, G. Faini, and V. Jeudy, Appl. Phys. Lett. 97, 243505 (2010). 